\title{
COASSIMETRIA, COCURTOSE E AS TAXAS DE RETORNO DAS AÇÕES: UMA ANÁLISE
} COM DADOS EM PAINEL

\section{FRANCISCO HENRIQUE FIGUEIREDO DE CASTRO JUNIOR}

Doutor em Administração pela Faculdade de Economia, Administração e Contabilidade da Universidade de São Paulo (USP). Professor do Programa de Mestrado em Contabilidade do Centro Universitário Fundação Escola de Comércio Álvares Penteado (Fecap). Avenida Liberdade, 532, Liberdade, São Paulo - SP - Brasil - CEP 01502-001

E-mail: henrique.castro@fecap.br

\section{CLAUDIA EMIKO YOSHINAGA}

Doutora em Administração pela Faculdade de Economia, Administração e Contabilidade da Universidade de São Paulo (USP). Professora da Escola de Administração de Empresas de São Paulo da Fundação Getulio Vargas (FGV-SP). Avenida Nove de Julho, 2.029, Bela Vista, São Paulo - SP - Brasil - CEP 01313-902 E-mail: claudia.yoshinaga@fgv.br 


\section{RESUMO}

Modelos de apreçamento de ativos têm sido um tema sob constante investigação em finanças. Desde o capital asset pricing model (CAPM) proposto por Sharpe (I964), tais modelos relacionam, geralmente de maneira linear, a taxa de retorno esperada de um ativo ou carteira de ativos com fatores de risco sistêmico. Esta pesquisa apresenta um teste de um modelo de apreçamento, com dados brasileiros, introduzindo em sua formulação fatores de risco baseados em comomentos estatísticos. O modelo proposto é uma extensão do CAPM original acrescido da coassimetria e da cocurtose entre as taxas de retorno das ações das empresas que compõem a amostra e as taxas de retorno da carteira de mercado. Os efeitos de outras variáveis, como o valor de mercado sobre valor contábil, a alavancagem financeira e um índice de negociabilidade em bolsa, serviram de variáveis de controle. A amostra foi composta de I79 empresas brasileiras não financeiras negociadas na BM\&FBovespa e com dados disponíveis entre os anos de 2003 a 2007. A metodologia consistiu em calcular os momentos sistêmicos anuais a partir de taxas de retornos semanais e em seguida testá-los em um modelo de apreçamento, a fim de verificar se há um prêmio pelo risco associado a cada uma dessas medidas de risco. Foi empregada a técnica de análise de dados em painel, estimada pelo método dos momentos generalizado (GMM). O emprego do GMM visa lidar com potenciais problemas de determinação simultânea e endogeneidade nos dados, evitando a ocorrência de viés nas estimações. Os resultados das estimações mostram que a relação das taxas de retorno dos ativos com a covariância e a cocurtose são estatisticamente significantes. Os resultados se mostraram robustos a especificações alternativas do modelo. O artigo contribui para a literatura por apresentar evidências empíricas brasileiras de que há um prêmio pelo risco associado aos momentos sistêmicos.

\section{PALAVRAS-CHAVE}

Coassimetria; Cocurtose; Taxas de retorno; Dados em painel; GMM. 


\section{INTRODUÇÃ}

Modelos de apreçamento de ativos têm sido um tema sob constante investigação em finanças. Desde o capital asset pricing model (CAPM) proposto por Sharpe (I964), tais modelos relacionam, geralmente de maneira linear, a taxa de retorno esperada de um ativo ou carteira de ativos com fatores de risco sistêmico. Dentre variados modelos propostos na literatura, encontram-se os sugeridos por Black (I972), Merton (I973), Kraus e Litzenberger (I976), Ross (I976), Homaifar e Graddy (I988), Jagannathan e Wang (I996), Fang e Lai (I997) e Harvey e Siddique (2000).

Tais modelos suscitaram inúmeros outros trabalhos que buscaram testar a validade de suas proposições. Os resultados encontrados, algumas vezes conflitantes entre si, estimularam pesquisadores a concentrar atenção também nas técnicas estatísticas empregadas. Black, Jensen e Scholes (I972), e Fama e MacBeth (I973) desenvolveram uma metodologia específica para testar modelos de apreçamento e lhe deram o nome de metodologia em duas passagens (two-pass methodology). De acordo com essa metodologia, o coeficiente beta de cada ativo é estimado, naquilo que vem a ser a primeira passagem, usando dados longitudinais. Os coeficientes estimados são então utilizados, numa segunda passagem, como regressores de uma equação linear que relaciona o prêmio pelo risco dos ativos (variável dependente) ao fator de risco (regressor) estimado na primeira passagem. Uma análise das metodologias mais comuns utilizadas nos testes dos modelos de apreçamento, assim como dos resultados alcançados, pode ser encontrada em Shanken (I992) e Kan e Zhang (I999a, I999b).

Além dos aspectos metodológicos envolvidos no teste de um modelo de apreçamento, diversas anomalias são comumente relatadas na literatura. Tais anomalias incluem principalmente os efeitos tamanho - relatado inicialmente por Banz (I98I) -, alavancagem - relatado por Bhandari (I988) -, e a razão entre valor contábil e valor de mercado - relatado por Stattman (I980). Essas e outras anomalias são amplamente discutidas por Fama e French (I992, I993).

A cada verificação de uma anomalia, a reputação do CAPM como um modelo de equilíbrio de preços de mercado era colocada em dúvida. As anomalias davam indícios de que havia variáveis que apresentavam forte relação com as taxas de retorno, mas que eram omitidas no modelo. Porém, apesar da intuição a respeito dessa relação, é senso comum, conforme Barone-Adesi, Gagliardini e Urga (2004), que apenas a persistência ao longo do tempo do sinal e da magnitude dos coeficientes associados a esses fatores dará à comunidade científica a segurança de que algum deles é um fator verdadeiramente associado à taxa de retorno dos ativos e percebido como um fator de risco pelos investidores. 
Ademais, a não normalidade da distribuição das taxas de retorno de ativos financeiros é algo também bastante documentado. Conforme Bekaert et al. (1998), mercados emergentes são especialmente caracterizados por esses problemas de não normalidade, tornando os modelos baseados no critério esperança-variância inadequados. Os autores afirmam que não é indicado tratar tais mercados da mesma maneira que são tratados os mercados desenvolvidos, uma vez que suas taxas de retorno apresentam distribuições com desvios substanciais do padrão da normalidade.

Portanto, da forma como foi concebido originalmente por Sharpe (I964), há evidências de que o CAPM pode não ser um modelo adequado, especialmente para o mercado brasileiro. Assim, esta pesquisa objetiva apresentar e testar uma extensão do modelo CAPM considerando outros fatores de risco além do beta (variância sistêmica) do modelo original. Esses fatores são baseados em momentos de ordem superior à variância, como a assimetria e a curtose. Esse modelo foi testado para uma amostra de ações de I79 empresas brasileiras não financeiras negociadas na Bolsa de Valores, Mercadorias e Futuros (BM\&FBovespa).

A metodologia consistiu em calcular os momentos sistêmicos anuais a partir de taxas de retornos semanais e em seguida testá-los em um modelo de apreçamento, a fim de verificar se há um prêmio pelo risco associado a cada uma dessas medidas de risco. Foi empregada a técnica de análise de dados em painel, estimada pelo método dos momentos generalizado (generalized method of moments - GMM). Os resultados indicaram que a formulação original do CAPM é incompleta e que há um prêmio pelo risco associado a outros fatores, como a curtose sistêmica (cocurtose). Diversas outras análises econométricas foram conduzidas a fim de garantir uma correta especificação dos modelos estimados, e os resultados apontaram na direção da robustez dos achados.

As principais contribuições desta pesquisa são:

- Este trabalho inova ao utilizar a metodologia de dados em painel estimado por GMM para testar um modelo de apreçamento baseado em momentos sistêmicos. O uso do painel de dados possibilita levar em consideração características das empresas, como o fato de que suas medidas de risco podem mudar ao longo do tempo. Em uma metodologia transversal (cross-section), a dimensão tempo seria desconsiderada. Além disso, o emprego do GMM visa lidar com potenciais problemas de determinação simultânea e endogeneidade nos dados, evitando a ocorrência de viés nas estimações.

- Este trabalho apresenta evidências empíricas brasileiras de que há um prêmio pelo risco associado aos momentos sistêmicos incluídos no modelo. Esse resultado se mostrou robusto a diferentes especificações, assim como à eventual presença de observações influentes. 
O restante do artigo está assim dividido: a seção 2 traz a revisão da literatura sobre o tema desta pesquisa; a seção 3 discute a base de dados empregada, os critérios de seleção da amostra e a metodologia utilizada no artigo; a seção 4 descreve e discute os resultados encontrados; e, por fim, a seção 5 apresenta as conclusões do trabalho.

\section{FUNDAMENTAÇÃO TEÓRICA}

Os momentos de variáveis aleatórias servem para caracterizar as distribuições de probabilidade. O primeiro e segundo momentos definem uma distribuição normal (média e variância). O terceiro momento é a assimetria e o quarto, a curtose. O modelo de Markowitz usava os dois primeiros, pois assumia normalidade. Este estudo não assume essa hipótese e usa outros momentos.

Modelos de apreçamento de ativos buscam exatamente relacionar os fatores de risco ao comportamento dos retornos dos ativos. Modelos parcimoniosos, como o CAPM, emergiram e ganharam popularidade rápida por sua simplicidade e facilidade de implementação. Contudo, conforme Harvey e Siddique (2000), o escrutínio pelo qual esse modelo passou nos últimos anos fez com que aumentasse a desconfiança de que os retornos esperados de ativos podem não ser explicados apenas por um único fator de risco, o beta do ativo.

Ao mesmo tempo que o CAPM passou a ser testado empiricamente, surgiram pesquisas que se interessavam pela possível influência de outros momentos no modelo. Os primeiros estudos, como o conduzido por Arditti (1967), buscavam relacionar o comportamento das taxas de retorno à assimetria em um modelo que contava ainda com a variância dos retornos. Os principais resultados permitiam concluir que o terceiro momento era uma medida estatisticamente significante e que momentos de quarta ordem em diante adicionavam pouca ou nenhuma informação na explicação do comportamento das taxas de retorno.

Kraus e Litzenberger (I976) testaram empiricamente a preferência do investidor pelo terceiro momento, usando o conceito de coassimetria em vez da assimetria total usada por Arditti (1967). Essa coassimetria recebeu o nome de assimetria sistêmica (não diversificável) em uma referência direta ao CAPM original que chama a covariância de risco sistêmico. Nesse sentido, o beta poderia ter sido chamado de variância sistêmica, uma vez que não era mais o único fator de risco existente no modelo. A extensão do CAPM proposta por Kraus e Litzenberger (I976) se mostrou empiricamente válida, uma vez que a assimetria sistêmica foi estatisticamente significante. Foi verificado também que os investidores, conforme esperado, tendem a ter aversão ao segundo 
comomento (covariância) e preferência pelo terceiro comomento (coassimetria) positivo quando a assimetria da carteira de mercado é positiva.

Friend e Westerfield (i980) refizeram a pesquisa de Kraus e Litzenberger (1976), desta vez incorporando à análise não apenas ações, mas também títulos de dívida negociados em bolsa, e trabalharam com uma carteira de mercado composta por esses dois tipos de ativo. Eles encontraram evidências, não totalmente conclusivas, de que investidores pagam algum prêmio por assimetria positiva. Singleton e Wingender (I986) mostraram que ativos com assimetria positiva em dado período podem apresentar tanto assimetria negativa como positiva em um período seguinte e vice-versa. Entre seus resultados, destaca-se também a verificação de que a assimetria é reduzida quando os ativos são combinados em carteiras.

Lim (I989) testou o modelo de Kraus e Litzenberger (I976) usando o método dos momentos generalizado (GMM) de Hansen (I982). Uma das vantagens desse método é que ele não impõe hipóteses acerca da distribuição de probabilidade das taxas de retorno dos ativos. Esse fato é especialmente interessante para este tipo de estudo, uma vez que não há uma distribuição de probabilidade consensual para taxas de retorno com coassimetria. Como resultado, Lim (ig89) encontrou evidências de que a assimetria sistêmica possui um preço.

A curtose não recebeu a mesma atenção que a assimetria em testes de modelos de apreçamento. A inclusão do quarto momento só foi feita por Homaifar e Graddy (I988), que derivaram um modelo de apreçamento incorporando a cocurtose ao modelo de Kraus e Litzenberger (1976). Entre os outros autores que testaram um modelo com quatro momentos, estão Fang e Lai (1997), Jondeau e Rockinger (2002, 2006), Galagedera, Henry e Silvapulle (2003) e Harvey et al. (2004). Com dados mensais de retornos de empresas brasileiras no período de 1990 a 2003 , Silva (2005) testou diversos modelos de apreçamento usando comomentos e os fatores de Fama e French (1992, I993). Seus resultados indicaram que o beta e a cocurtose eram significantes em quase todas as suas estimações. Silva (2005) empregou a abordagem de regressão tradicional, além de agrupar as ações em carteiras. Porém, por causa das características do mercado brasileiro, essa decisão só permitiu a criação de cinco delas. Segundo Saito e Bueno (2007), talvez seja desnecessário empregar carteiras em vez de ativos individuais especialmente no Brasil, onde há limitações de tamanho das amostras.

A questão da periodicidade ou do intervalo com que os dados amostrais são calculados também é um fator importante a ser considerado nesta pesquisa. Os dados de retorno, que serão calculados com base nas observações dos preços, podem ser coletados com periodicidade diária, semanal, mensal ou qualquer outra periodicidade desejada. Porém, há estudos que mostram que os resultados 
podem ser consideravelmente afetados por essa escolha. Fogler e Radcliffe (I974) ilustram que a assimetria é mais sensível que a média e o desvio padrão quando diferentes intervalos de tempo são usados para seu cálculo. Com uma amostra de retornos anuais de carteiras de ativos no período de I954 a I963, encontraram que a série das taxas de retorno era positivamente assimétrica. Contudo, quando a assimetria foi calculada com base em intervalos menores (semestrais e trimestrais), chegava-se à conclusão oposta. O mesmo resultado foi encontrado para o índice Standard and Poor's nos anos de 1948 a I969.

Smith (I978) também encontrou diferenças significativas quando diferentes intervalos foram usados na construção da série de retornos. O CAPM foi testado em diferentes carteiras com taxas de retorno construídas a partir de intervalos distintos, e, segundo seus achados, os valores de alfa e beta foram sensíveis a essa escolha até ele separar as carteiras com base no risco sistêmico. Quando isso foi feito, as estimativas de beta das carteiras aumentaram com o aumento do intervalo para o grupo de ações de maior beta e diminuíram com o aumento do intervalo para o grupo de ações de menor beta.

Conforme Galagedera, Henry e Silvapulle (2003), a assimetria e a curtose das distribuições das taxas de retorno são mais elevadas em dados de alta frequência, razão pela qual esses autores decidiram usar dados diários em sua pesquisa. Jondeau e Rockinger (2003), na estimação da volatilidade, assimetria e curtose condicionais, fizeram uso de dados diários. Assim também o fizeram Brooks et al. (2005), que verificaram em seus dados que a curtose diminui com a agregação dos retornos.

Entretanto, há também exemplos de aplicação e teste de modelos de apreçamento com taxas de retorno calculadas com intervalos de frequência mais baixa. Fang e Lai (I997), por exemplo, usaram dados mensais e não fizeram nenhuma referência às mudanças nas estimativas dos momentos causadas por essa decisão. O mesmo comportamento foi adotado por Harvey e Siddique (2000) para seu modelo condicional de três momentos. Silva (2005) também utilizou taxas de retorno mensais. Esses exemplos ilustram que a decisão pela periodicidade de coleta dos preços dos ativos não é consensual na literatura. O que alguns autores verificaram empiricamente Hawawini (I980) mostrou analiticamente que a assimetria da taxa de retorno composta continuamente é sensível à escolha do tamanho do intervalo com que essa taxa de retorno é calculada. Além disso, mostrou também que essa sensibilidade será maior quanto maior for a ordem do momento. Lau e Wingender (I989) mostraram que, quando os retornos são calculados na forma discreta, a periodicidade com que os retornos são calculados também exerce efeitos sobre os momentos. Além disso, é importante notar que séries financeiras não são, em geral, estacionárias. A falta de estacionariedade 
provoca a necessidade de tratar as séries financeiras como séries que variam no tempo, e seus momentos devem ser estimados levando-se em conta esse fato.

De acordo com Bekaert et al. (I998), a análise por esperança e variância tradicional no conceito de Markowitz (I952, I959) é especialmente problemática em mercados emergentes. Em um estudo específíco para esses mercados, os autores constataram que os ativos transacionados apresentam assimetria e curtose significativas em seus retornos, implicando que é razoável acreditar que os investidores devam ter preferências por esses momentos. Além disso, verificaram também que esses momentos variam significativamente ao longo do tempo, fato que sugere a necessidade de usar uma metodologia que leve em consideração esse tipo de característica. A iniciativa, nesta pesquisa, de utilizar dados em painel decorre dessa necessidade.

\section{METODOLOGIA}

Um dos propósitos desta pesquisa é apresentar uma inovação na forma de testar um modelo de apreçamento. A abordagem proposta busca evitar alguns dos problemas enfrentados por estudos anteriores, especialmente no que se refere à não estabilidade das medidas de risco ao longo do tempo.

A abordagem é conduzida em duas etapas: na primeira delas, descrita a seguir, é utilizado um modelo de fatores para a estimação dos comomentos; em seguida, estes são utilizados no teste do modelo de apreçamento empregando o painel de dados.

\subsection{MODELOS DE FATORES}

O principal resultado teórico do CAPM é o estabelecimento da relação entre o prêmio pelo risco de determinado ativo $i$ (ou carteira de ativos), $E\left[r_{i}\right]-r_{f}$, e seu risco sistêmico, $\beta_{i}$, conforme a equação a seguir:

$$
E\left[r_{i}\right]-r_{f}=\beta_{i}\left(E\left[r_{M}\right]-r_{f}\right)
$$

tal que $E\left[r_{i}\right]$ é a esperança da taxa de retorno do ativo ou carteira $i ; r_{f}$, a taxa de retorno do ativo livre de risco; e $E\left[r_{M}\right]$, a esperança da taxa de retorno da carteira de mercado.

Se for definido um $\alpha_{i}$, tal que

$$
\alpha_{i}=E\left[r_{i}\right]-r_{f}-\beta_{i}\left(E\left[r_{M}\right]-r_{f}\right)
$$


então, pela Equação (I), $\alpha_{i}=0$ para qualquer ativo. Basta, portanto, verificar se isso é empiricamente verdadeiro. O teste formal do CAPM, conforme sugerido por Black, Jensen e Scholes (I972), é testar se, para um conjunto de empresas, a equação

$$
r_{i}-r_{f} i=\theta_{\mathrm{o}}+\theta_{\mathrm{I}} \beta_{i}+\varepsilon_{i}
$$

apresenta $\theta_{\mathrm{o}}=\mathrm{o}$ e $\theta_{\mathrm{I}}$ igual ao excesso de retorno da carteira de mercado. Nessa equação, $r_{i}$ é um vetor de dimensão das taxas de retorno das $\mathrm{N}$ empresas; $i$, um vetor de dimensão $N \times$ I de I's; $\beta_{i}$, o vetor $N \times$ I de medidas de risco baseadas nas covariâncias das taxas de retorno das empresas com a taxa de retorno da carteira de mercado; e $\varepsilon_{i}$, o vetor de dimensão $N \times$ I de erros, satisfazendo $E\left[\left(\varepsilon_{i} \mid r_{M}, r_{f}\right)\right]=0$.

As pesquisas que apontam na direção de uma deficiência do CAPM, como as apresentadas na fundamentação teórica, sugerem que há fatores de risco não considerados em sua formulação original. Uma das proposições é que fatores de risco associados aos comomentos de terceira e quarta ordens são potencialmente relevantes. A estimação desses comomentos se dá com o uso de modelos lineares nos parâmetros. Desde Kraus e Litzenberger (I976), o modelo de mercado quadrático é empregado na estimação das coassimetrias entre as séries de taxas de retorno dos ativos e da carteira de mercado. O processo gerador de dados para as séries de taxas de retorno, segundo esse modelo, é dado pela seguinte equação:

$$
r_{i}-r_{f} i=\alpha_{i}+\beta_{i}\left(r_{M}-r_{f}\right)+\gamma_{i}\left(r_{M}-r_{f}\right)^{2}+\varepsilon_{i}
$$

tal que $\alpha_{i}$ é um vetor de dimensão $N \times$ I de interceptos; $\beta_{i}$, o vetor de dimensão $N \times$ I de covariâncias; $\gamma_{i}$, o vetor de dimensão $N \times$ I de coassimetrias; e $\varepsilon_{i}$, o vetor de dimensão $N \times$ I de erros, satisfazendo $E\left[\left(\varepsilon_{i} \mid r_{M}, r_{f}\right)\right]=0$.

Conforme Barone-Adesi, Gagliardini e Urga (2004), a motivação para incluir o quadrado do excesso de retorno da carteira de mercado é considerar a coassimetria entre a taxa de retorno de cada ativo e a taxa de retorno da carteira de mercado. Esses autores verificaram que os resíduos da regressão entre o excesso de retorno de alguns ativos contra uma constante e o excesso de retorno da carteira de mercado tendem a ser positivamente (negativamente) correlacionados com o excesso de retorno ao quadrado da carteira de mercado. Tais ativos apresentaram tendência a ter taxas de retorno mais altas (mais baixas) quando o mercado exibia taxas de retorno absolutas mais altas. Constatou-se que tais ativos apresentavam coassimetria positiva (negativa) com o mercado.

Assim, além do clássico coeficiente $\beta$ do CAPM, a coassimetria é, também, um fator de risco. O teste empírico do modelo de mercado quadrático é por meio da seguinte equação: 


$$
r_{i}-r_{f} i=\theta_{\circ}+\theta_{\mathrm{I}} \beta_{i}+\theta_{2} \gamma_{i}+\varepsilon_{i}
$$

tal que $\theta_{\circ}=\circ$ e os demais coeficientes $\theta_{i}$ serão significativamente diferentes de zero quando o vetor de comomentos aos quais estão relacionados for relevante para o modelo. Seguindo a mesma intuição do modelo de mercado quadrático, o modelo de mercado cúbico insere outro comomento, a cocurtose entre as séries de taxas de retorno dos ativos e da carteira de mercado. O modelo de mercado cúbico é dado pela seguinte equação:

$$
r_{i}-r_{f} i=\alpha_{i}+\beta_{i}\left(r_{M}-r_{f}\right)+\gamma_{i}\left(r_{M}-r_{f}\right)^{2}+\delta_{i}\left(r_{M}-r_{f}\right)^{3}+\varepsilon_{i}
$$

tal que, além das mesmas variáveis anteriormente descritas, $\delta_{i}$ é o vetor de dimensão $N \times$ I de cocurtoses. A equação a seguir permite a condução de um teste empírico para o modelo de mercado cúbico.

$$
r_{i}-r_{f} i=\theta_{\mathrm{o}}+\theta_{\mathrm{I}} \beta_{i}+\theta_{2} \gamma_{i}+\theta_{3} \delta_{i}+\varepsilon_{i}
$$

tal que $\theta_{\circ}=$ o e os demais coeficientes $\theta_{i}$ serão significativamente diferentes de zero quando o vetor de comomentos aos quais estão relacionados for relevante para o modelo.

\subsection{POPULAÇÃO E AMOSTRA}

Fazem parte da população de interesse desta pesquisa todas as empresas com ações regularmente negociadas na BM\&FBovespa no período de $\mathrm{I}^{\circ}$ de janeiro de 2003 a 3I de dezembro de 2007 . A seleção de um período específico para coleta dos dados por si só já constitui um critério de seleção de amostra. Nesse caso, pode ainda criar um viés de sobrevivência à amostra selecionada, uma vez que apenas as empresas negociadas nesse período poderão fazer parte dela. Contudo, conforme Singleton e Wingender (I986), os resultados qualitativos da análise sofrem pouca interferência por conta desse fato.

A amostra foi composta por I79 empresas não financeiras. A fim de chegar a esse número, diversos filtros foram utilizados. Primeiramente, foram identificadas todas as empresas cujas ações tiveram data de início de negociação anterior a $1^{\circ}$ de janeiro de 2006 e data de último negócio posterior a 3I de dezembro de 2004. Dessa forma, foram descartadas as empresas cujas ações apresentaram negociação por um período inferior a dois anos. Em seguida, as empresas com baixo índice de negociação em bolsa foram excluídas da amostra: foi adotado como critério de corte o valor mínimo de o,or em cada ano. A justificativa para o 
uso desse critério é que ações de empresas com baixa negociabilidade implicam séries com muitos dados faltantes, tornando complexa a determinação do seu preço correto.

Para cada empresa, foi escolhida apenas uma única classe de ação: aquela com maior volume de negociação no período analisado. Essa decisão foi tomada porque na análise de dados foram utilizadas informações contábeis das empresas. Como uma mesma empresa podia ter ações de diferentes classes, mas seus dados contábeis seriam os mesmos, isso causaria uma inconsistência. Em seguida, coletaram-se dados de preços semanais das ações da amostra. Algumas empresas apresentaram, para alguns anos, pouca frequência de dados semanais. Aquelas que não apresentaram pelo menos dois anos com o mínimo de 40 semanas de dados de preço em cada um deles foram totalmente excluídas da amostra. Das restantes, os anos com menos de 40 observações de dados semanais também foram eliminados. Ou seja, uma empresa para fazer parte da amostra deveria ter pelo menos 40 ou mais observações de preço semanais em pelo menos dois dos anos. Isso caracteriza a amostra como um painel desbalanceado. Essa decisão minimiza o viés de sobrevivência da amostra, uma vez que foi aceita a possibilidade de a empresa ter encerrado, ou mesmo iniciado, sua atividade em bolsa durante o período amostral. Todos os dados foram obtidos da base de dados do sistema de informações da Economática. Utilizou-se também a classificação por setor de atuação de acordo com as 20 categorias definidas por ela. Na amostra, todos esses setores, exceto o de empresas financeiras, estão representados.

\section{3 INVESTIGAÇÃO EMPÍRICA}

A análise de dados em painel permite o estudo econométrico ao longo do tempo de unidades básicas de estudo dispostas em cortes transversais. Nesta pesquisa, a unidade básica de estudo é representada por empresas, observadas em diferentes instantes do tempo. A vantagem no uso dessa técnica é, em primeiro lugar, permitir a observação de relações dinâmicas, algo que não é alcançado apenas com dados transversais. Em segundo lugar, o painel permite o controle da heterogeneidade não observada nos dados transversais. Outro benefício do uso dessa abordagem está relacionado à possibilidade de utilização de uma base de dados maior do que seria possível apenas com dados transversais. Essa base de dados maior, segundo Baltagi $(2005,2006)$, permite uma maior variabilidade e uma menor colinearidade entre as variáveis estudadas. O conjunto de dados desta pesquisa caracteriza-se por ser um painel do tipo curto, ou seja, há disponibilidade de uma quantidade grande de observações transversais (empresas) em 
poucos instantes longitudinais (tempo). Outra característica da amostra estudada é que ela forma um painel desbalanceado, ou seja, não há dados disponíveis para todas as empresas em todos os anos.

A relação entre as variáveis de interesse da pesquisa pode ser representada conforme a equação linear a seguir:

$$
y_{i, t}=\theta_{\mathrm{o}}+\theta^{\mathrm{T}} \Theta_{i, t}+\omega^{\mathrm{T}} \Omega_{i, t}+\varepsilon_{i, t}
$$

tal que, para cada empresa $i$ em determinado ano $t$, observou-se um conjunto de $k_{\mathrm{I}}$ comomentos $\Theta_{i, t}$, um vetor de dimensão $k_{\mathrm{I}} \times$ I. O vetor $\theta^{\mathrm{T}}$ é o vetor de dimensão $k_{\mathrm{I}} \times$ I de parâmetros associados a cada comomento, transposto. O conjunto das $k_{2}$ variáveis de controle está representado no vetor $\Omega_{i, t}$. O vetor $\omega^{\mathrm{T}}$ de dimensão $k_{2} \times \mathrm{I}$, transposto, é o conjunto de parâmetros das variáveis de controle. O termo de erro, $\varepsilon_{i, t}$, não deve, por hipótese, se correlacionar contemporaneamente com os regressores; e a variável de resposta, $\gamma_{i, t}$, é o prêmio pelo risco da empresa $i$ no instante de tempo $t$.

Ao estabelecer um modelo de relacionamento estatístico entre variáveis, cabe destacar a potencial existência de endogeneidade das variáveis, ou seja, a existência de correlação entre os regressores e os termos de erro do modelo. Segundo Wooldridge (2002, p. 50), a presença de variáveis com problemas de endogeneidade leva à estimação de parâmetros inconsistentes no modelo e suas principais causas são: I. variáveis omitidas: não inclusão de variáveis no modelo de regressão que sejam correlacionadas com os regressores e a variável dependente; 2. erros de medida dos regressores: é possível que a variável de interesse não seja observável, tendo-se à disposição uma medida imperfeita que pode não apresentar correlação com a variável de resposta da mesma forma que o seu valor real, além de eventualmente ser correlacionada com o erro; e 3. simultaneidade: variáveis regressoras podem ser simultaneamente determinadas com a variável dependente.

Com o objetivo de reduzir problemas de endogeneidade, foram inseridas no modelo de regressão algumas variáveis de controle sugeridas por estudos teóricos e empíricos anteriores, dummies de tempo e variáveis instrumentais. Conforme Wooldridge (2002, p. 83-84), um regressor, para ser considerado uma variável instrumental, deve ser: não correlacionado com o erro do modelo a ser estimado e parcialmente correlacionado com a variável endógena.

Tradicionalmente, modelos com dados em painel utilizam os métodos de estimação de dados agrupados (pooled ordinary least squares - Pols), efeitos aleatórios (EA) e efeitos fixos (EF). Embora estes sejam os métodos tradicionalmente utilizados para a estimação com dados em painel, para que suas estimativas 
sejam consistentes é preciso que haja a exogeneidade estrita dos regressores. Isso significa que o termo de erro do modelo deve ser não correlacionado com as variáveis explicativas em todos os instantes do tempo. Para verificar a condição de exogeneidade estrita dos regressores e atestar a validade da estimação por efeitos aleatórios e fixos, Wooldridge (2002, p. 285) propõe dois testes de exogeneidade estrita: o primeiro baseado no estimador de primeira diferença e o segundo no estimador de EF. A hipótese nula do teste de exogeneidade estrita usando primeiras diferenças é: $H_{0}: \xi=0$, conforme Equação (8).

$$
\Delta \gamma_{i, t}=\Delta \mathrm{x}_{i, t} \mathcal{v}+\mathrm{w}_{i, t} \xi+\Delta \varepsilon_{i, t}, \quad t=2, \ldots, \mathrm{T}
$$

tal que $\gamma_{i, t}$ é o prêmio pelo risco da empresa $i$ no instante $t$; $\mathrm{x}_{i, t}$, o vetor de comomentos e variáveis de controle, exceto dummies; $\mathrm{w}_{i, t}$, todo o conjunto de regressores da Equação (7); $\varepsilon_{i, t}$, o termo de erro associado ao ativo i no instante t; e $\Delta$, o operador matemático da diferença entre as variáveis nos instantes $t$ e $t-\mathrm{I}$.

Já no teste de exogeneidade estrita usando efeitos fixos, a hipótese nula é $H_{\mathrm{o}}$ : $\xi=0$, conforme a Equação (9).

$$
\gamma_{i, t}=\mathrm{x}_{i, t} v+\mathrm{w}_{i, t} \zeta+\eta_{i}+v_{i, t}, \quad t=\mathrm{I}, \ldots, \mathrm{T}-\mathrm{I}
$$

tal que $\gamma_{i, t}$ é o prêmio pelo risco da empresa $i$ no instante $t ; \mathrm{x}_{i, t}$, o vetor de comomentos e variáveis de controle, exceto dummies; $\mathrm{w}_{i, t}$, todo o conjunto de regressores da Equação (7); $\eta_{i}$, a heterogeneidade não observada do ativo $i$; e $v_{i, t}$, o erro aleatório do ativo $i$ no instante $t$.

Esses dois testes são fundamentais para justificar a utilização dos métodos tradicionais de estimação com dados em painel. Apesar de os estudos em geral iniciarem a apresentação de seus resultados com modelos estimados por Pols e prosseguirem com EA e EF, estes apresentam resultados que são consistentes apenas na hipótese de exogeneidade estrita dos regressores. Quando esse não for o caso, será necessário adotar um método de estimação como o método dos momentos generalizado (GMM) proposto por Arellano e Bond (I99), Arellano e Bover (I995) e Blundell e Bond (I998) a fim de lidar com possíveis problemas de simultaneidade e causalidade reversa.

Quando os regressores são correlacionados com os termos de erro, a ideia é substituí-los por estimativas de seus valores que não o sejam. Essa abordagem é chamada de variáveis instrumentais e os regressores substitutos são denominados instrumentos. Para que uma variável possa ser utilizada como instrumento, ela deve atender a dois requisitos: I. não deve ser correlacionada com as variáveis explicativas endógenas; e 2. o instrumento não deve ser corre- 
lacionado com o termo de erro, ou seja, não deve apresentar o mesmo problema que a variável substituída. Normalmente as defasagens das variáveis regressoras são utilizadas como instrumentos, ou seja, para instrumentar $x_{i, t}$, usam-se $x_{i, t-1}, x_{i, t-2}, \ldots, x_{i, t-n}$.

\subsubsection{GMM em diferenças}

Sejam as seguintes equações:

$$
\begin{gathered}
Y_{i, t}=\beta^{\mathrm{T}} \mathrm{z}_{i, t}+\varepsilon_{i, t}, \\
\varepsilon_{i, t}=\eta_{i}+v_{i, t}, \\
E\left[\eta_{i}\right]=E\left[v_{i, t}\right]=E\left[\eta_{i} v_{i, t}\right]=0
\end{gathered}
$$

O termo de erro, $\varepsilon_{i, t}$, possui dois componentes ortogonais: o efeito não observado específico para cada empresa, $\eta_{i}$, e o termo de erro aleatório, $v_{i, t}$ O método conhecido como GMM em diferenças (GMM-Dif), desenvolvido por Arellano e Bond (I99I), transforma todos os regressores ao extrair as primeiras diferenças das variáveis em relação aos seus valores passados. Aplicando essa transformação à Equação (Io), obtém-se a equação do GMM-Dif:

$$
\Delta \gamma_{i, t}=\beta^{\mathrm{T}} \Delta z_{i, t}+\Delta \mathbf{v}_{i, t}
$$

tal que $\Delta \gamma_{i, t} \stackrel{\text { def }}{=} \gamma_{i, t}-\gamma_{i, t-\mathrm{I}}, \Delta z_{i, t} \stackrel{\text { def }}{=} z_{i, t}-z_{i, t-\mathrm{I}}$ e $\Delta \varepsilon_{i, t} \stackrel{\text { def }}{=} \varepsilon_{i, t}-\varepsilon_{i, t-\mathrm{I}}$ Esse procedimento, consequentemente, elimina a heterogeneidade não observada, uma vez que esta é invariante no tempo $\left(\Delta \eta_{i}=0\right)$. A transformação de primeira diferença tem a vantagem de dispensar qualquer suposição acerca da correlação entre $\eta_{i}$ e $z_{i, t}$.

Essa vantagem, entretanto, pode levar a uma redução considerável na quantidade de pontos caso o painel tenha períodos sem observação no meio da série ou empresas com poucas observações. Considerando que a amostra é um painel desbalanceado, o cálculo de diferenças pode reduzir drasticamente a quantidade de observações. Para lidar com esse problema, foram utilizadas as transformações de desvios ortogonais, conforme proposto por Arellano e Bover (I995). Para verificar a validade dos instrumentos utilizados na estimação por GMM, deve ser aplicado o teste de autocorrelação de Arellano e Bond (I99I) nos termos de erro $v_{i, t}$ O termo de erro $\varepsilon_{i, t}$ é presumidamente autocorrelacionado, pois ele contém o efeito fixo não observado $\eta_{i}$, e o estimador GMM foi construído para eliminar 
esse problema. No entanto, $v_{i, t}$ apresentará autocorrelação de primeira ordem se $\mathrm{z}_{i, t-2}$ for endógeno a $v_{i, t-\mathrm{I}} \mathrm{e}$, consequentemente, a $\Delta \varepsilon_{i, t}=v_{i, t}-v_{i, t-\mathrm{I}}$. Conforme Roodman (2009), isso tornaria o termo $\mathrm{z}_{i, t-2} \mathrm{um}$ instrumento inválido. Caso isso ocorra, é necessário restringir a defasagem dos instrumentos para 3 ou mais períodos. Se for encontrada uma autocorrelação de ordem 2, esse processo terá que incluir defasagens maiores ainda e assim por diante.

Considerando que os estimadores de GMM-Dif são gerados a partir das primeiras diferenças, seus resíduos tendem a ser autocorrelacionados e por isso devem ser aplicados testes de autocorrelação. Como $\Delta v_{i, t}$ é matematicamente relacionado a $\Delta v_{i, t-\mathrm{I}}$ por compartilharem o termo $v_{i, t-\mathrm{I}}$, espera-se uma autocorrelação de primeira ordem negativa (estatística $m_{\mathrm{I}}$ ). Além da autocorrelação de primeira ordem nas diferenças, é preciso dar atenção à autocorrelação de segunda ordem $\left(m_{2}\right)$ : espera-se encontrar $m_{2}$ não significante caso $\Delta \mathrm{v}_{i, t}$ seja não autocorrelacionado de segunda ordem. Resumidamente, espera-se rejeitar a hipótese nula na primeira ordem, mas não na segunda ordem em diante, isto é, deseja-se encontrar um valor negativo e significante para $m_{\mathrm{I}}$ e não significante para $m_{2}$.

Outro teste de especificação importante é o de sobreidentificação. Uma vez que a quantidade de instrumentos utilizados supera a quantidade de parâmetros a serem estimados, aplica-se o teste de restrições de Sargan (I958) para verificar a validade do conjunto de instrumentos. A hipótese nula do teste é que as variáveis instrumentais e os resíduos não são correlacionados e os instrumentos são válidos, podendo ser utilizados no modelo. Esse teste é, entretanto, inconsistente quando se suspeita da não esfericidade nos erros, conforme Roodman (2009). Nesse caso, recomenda-se o teste de Hansen (1982), que calcula a estatística para uma estimativa de dois estágios. Baum, Schaffer e Stillman (2003) afirmam que a estatística do teste de Sargan (I958) é um caso específico do J de Hansen (I982). Para uma situação de homoscedasticidade condicional, ambas apresentariam o mesmo valor.

\subsubsection{GMM sistêmico}

O modelo de Blundell e Bond (I998) é um aperfeiçoamento da versão do GMM em diferenças com a adição da premissa de que as primeiras diferenças das variáveis instrumentais não são correlacionadas com os erros, ou seja:

$$
E\left[\Delta z_{i, t-1} \varepsilon_{i, t}\right]=0 \Rightarrow E\left[\Delta z_{i, t-1}\left(v_{i, t}+\eta_{i}\right)\right]=0
$$

Essa restrição adicional de não correlação entre $\Delta z_{i, t-1}$ e $v_{i, t}$ permite a inclusão de mais instrumentos ( $\Delta \mathrm{z}_{i, t-1}$ e as defasagens em nível), o que consequente- 
mente melhora a eficiência da estimação. Esse modelo, conhecido como GMM sistêmico (GMM-Sis), baseia-se em um sistema de equações, em que os instrumentos usados nas equações em níveis são as primeiras diferenças defasadas das séries e os instrumentos usados nas equações em primeiras diferenças são os níveis defasados das séries (BOND; HOEFFLER; TEMPLE, 200I).

Os mesmos testes de sobreidentificação usados no GMM-Dif são empregados no GMM-Sis. Os testes de Sargan/Hansen avaliam todo o conjunto de restrições, mas o seu poder se reduz à medida que a proporção entre a quantidade de instrumentos e de observações aumenta. Há um problema adicional quando existem suspeitas iniciais quanto à validade dos instrumentos empregados (BAUM; SCHAFFER; STILLMAN, 2003).

$\mathrm{Na}$ análise de diagnóstico do modelo, pode ser aplicado o teste DIF-Hansen. Esse procedimento baseia-se na diferença entre as estatísticas dos testes de Hansen para um mesmo modelo, estimado por GMM-Sis e por GMM-Dif. A hipótese nula, se não rejeitada, indica que as condições adicionais para o uso do GMM-Sis são válidas. Os estimadores GMM-Dif e GMM-Sis utilizam as condições de momentos para estimar os parâmetros, consistente e eficientemente, de acordo com duas variações: um ou dois estágios. O estimador em dois estágios é assintoticamente mais eficiente que o estimador de um estágio, mas os erros padrão resultantes podem ser enviesados para baixo (ARELLANO; BOND, I99I; BLUNDELL; BOND, I998). Como consequência, os resultados podem ser imprecisos, especialmente para amostras finitas e com grande quantidade de instrumentos. Contudo, para a estimação do painel pelo estimador GMM-Sis de dois estágios, utilizou-se a correção proposta por Windmeijer (2005) na matriz de variâncias para tratar a heteroscedasticidade e resultar em estimativas corrigidas dos erros padrão em amostras finitas. A partir de resultados de simulações, Windmeijer (2005) demonstrou que a correção proposta tornava as estimativas em dois estágios mais eficientes que as estimativas em um estágio mesmo em amostras finitas.

\subsection{ALTERNATIVAS ROBUSTAS DE ANÁLISE}

Uma preocupação recorrente em estudos empíricos diz respeito às observações ditas extremas (outliers). Para tratar esse problema, utilizaram-se variáveis "winsorizadas". Nesta pesquisa, $2 \%$ dos valores das variáveis foram considerados extremos, sendo I\% na parte inferior e I\% na parte superior. Barnett e Lewis (I994) apresentam essa técnica detalhadamente e ressaltam sua importância pelo fato de que, ao contrário de outras técnicas que excluem da amostra certas observações consideradas extremas, a "winsorização" não joga 
fora nenhuma observação, apenas a torna menos extrema. É esperado que os resultados das estimações obtidos após o processo de "winsorização" não sejam decisivamente alterados.

\subsection{DEFINIÇÃO OPERACIONAL DAS VARIÁVEIS}

A seguir, estão descritas as definições operacionais das variáveis utilizadas nesta pesquisa.

\subsubsection{Prêmio pelo risco das ações}

A abordagem econométrica utilizada nesta pesquisa se dá em duas etapas, conforme metodologia proposta por Black, Jensen e Scholes (I972) e Fama e MacBeth (I973). Nessas duas etapas, usa-se como variável dependente o prêmio pelo risco das ações que fazem parte da amostra. Esse prêmio é definido como a diferença entre a taxa de retorno composta continuamente da ação e a taxa de retorno composta continuamente do ativo livre de risco. O CDI foi usado como proxy para a taxa de retorno do ativo livre de risco. O Ibovespa foi utilizado como proxy para a carteira de mercado.

\subsubsection{Tamanho da empresa}

Uma das variáveis frequentemente encontradas em testes de modelos de apreçamento é o tamanho da empresa. Essa variável, que assume o papel de variável de controle, tem por finalidade expurgar da relação de regressão os efeitos do tamanho das empresas sobre as taxas de retorno observadas. O chamado efeito tamanho foi inicialmente verificado na pesquisa de Banz (I98I). Nela, o autor verificou que empresas menores tiveram retornos ajustados ao risco maiores que empresas maiores, levantando a questão de o CAPM ser um modelo mal especificado. Conforme Berk (I995), ainda não é possível explicar teoricamente esse efeito. Exatamente por isso, esse efeito, quando verificado, é tratado como uma anomalia. Na verdade, nas pesquisas conduzidas, nunca foi possível dizer que o tamanho seria um fator importante, porém ausente no modelo, ou se ele era apenas uma variável capturando o efeito de alguma outra, ainda desconhecida, mas correlacionada a esta.

Nesta pesquisa, o tamanho da empresa foi definido como o logaritmo natural da receita líquida da empresa, $\ln R L_{i, t}$. As receitas líquidas foram obtidas, sempre que possível, por meio dos demonstrativos consolidados dos resultados dos exercícios. 


\subsubsection{Valor de mercado sobre valor contábil das ações}

Essa variável de controle também é comumente utilizada em testes de modelos de apreçamento. Na literatura, geralmente é chamada de book-to-market equity (BE/ME), que seria o inverso do valor de mercado sobre o valor contábil (VM/VC). Stattman (I980), Rosenberg, Reid e Lanstein (I985) e Fama e French (I992) encontraram evidências do impacto dessa variável sobre a taxa de retorno das ações, mesmo controlando pelo beta. De acordo com esses estudos, quanto maior o BE/ME, maior a taxa de retorno verificada.

Nesta pesquisa, o índice valor de mercado sobre valor contábil $\left(V M V C_{i, t}\right)$ das ações para cada ano foi calculado dividindo o valor de mercado da empresa pelo seu patrimônio líquido $(P L)$ no fechamento do ano.

\subsubsection{Negociabilidade}

O índice de negociabilidade, $N E G_{i, t}$, adotado foi calculado para cada empresa, conforme a seguinte equação:

$$
N E G_{i, t}=\mathrm{IOO} \times \frac{d_{i, t}}{D_{t}} \sqrt{\frac{n_{i, t}}{N_{t}} \times \frac{v_{i, t}}{V_{t}}}
$$

tal que $d_{i, t}$ é o número de dias em que houve pelo menos um negócio com a ação da empresa i dentro do ano $t$; $D_{t}$, o número total de dias do ano $t ; n_{i, t}$, o número de negócios com a ação da empresa i no ano t; $N_{t}$, o número de negócios com todas a ações no ano $t ; v_{i, t}$, o volume em dinheiro dos negócios realizados com a ação da empresa i no ano t; e $V_{t}$, o volume em dinheiro dos negócios realizados com todas as ações no ano $t$.

\subsubsection{Alavancagem}

O efeito alavancagem é também frequentemente verificado como uma anomalia em testes de modelos de apreçamento. Esse efeito foi apontado inicialmente por Bhandari (I988). Conforme Fama e French (I992), é plausível que a alavancagem esteja associada ao risco e ao retorno esperado dos ativos, mas o beta do CAPM deveria capturar esse tipo de risco. Os resultados de pesquisas como a de Bhandari (I988) destacam que o retorno esperado das ações é positivamente relacionado com a alavancagem, estando a alavancagem líquida 
dos efeitos tanto do beta da empresa como do seu tamanho. Nesta pesquisa, a alavancagem, $D B V M_{i, t}$, foi medida como a dívida bruta sobre valor de mercado das ações da empresa. A dívida bruta corresponde à soma dos financiamentos de curto e longo prazos, assim como as debêntures de curto e longo prazos emitidas pelas empresas.

\subsubsection{Setor de atividade}

Dezenove dos vinte setores de atividade, conforme classificação da Economática, estão representados neste estudo. Cada setor corresponde a uma variável dummy, do tipo binária, que assume o valor i quando a empresa pertencer a um determinado setor ou o quando pertencer a quaisquer dos outros I8 setores. Para efeito da estimação dos modelos, empresas pertencentes ao setor financeiro e de seguros foram excluídas da amostra. Tais empresas apresentam características muito particulares que as distinguem das empresas dos demais setores.

\subsubsection{Dummies de ano}

Essas variáveis também são do tipo binária, assumindo valor I quando corresponder ao t-ésimo ano ou o quando corresponder a quaisquer dos outros anos. O objetivo de inserir esse tipo de variável no modelo é tentar capturar eventuais choques macroeconômicos que afetaram todas as empresas da amostra no intervalo de tempo estudado.

\section{RESULTADOS}

\subsection{ESTATÍSTICAS DESCRITIVAS}

O índice de mercado utilizado nesta pesquisa foi o Ibovespa. As taxas de retorno semanais dessa carteira (para cada ano e para todo o período) foram calculadas, e suas estatísticas descritivas são apresentadas na Tabela I. É possível perceber que a carteira de mercado é assimétrica à esquerda em todos os períodos. A curtose ora é maior que 3 (valor de referência por ser a curtose de uma distribuição normal), ora é menor. As taxas de retorno semanais das empresas que compõem a amostra são, em sua maioria, assimétricas à direita: $72 \%$ das empresas têm assimetria positiva. 


\section{TABELA I}

ESTATÍSTICAS DESCRITIVAS DA CARTEIRA DE MERCADO

\begin{tabular}{cccccccc}
\hline ANO & MÉDIA & DP & ASSIM. & CURT. & $1^{\circ}$ QT. & MED. & $3^{\circ}$ QT. \\
\hline 2003 & 0,0083 & 0,0345 & $-0,6928$ & 3,0448 & $-0,0095$ & 0,0144 & 0,0321 \\
\hline 2004 & 0,0006 & 0,0378 & $-0,1584$ & 2,8731 & $-0,0260$ & 0,0033 & 0,0222 \\
\hline 2005 & 0,0014 & 0,0331 & $-0,3996$ & 2,4865 & $-0,0182$ & 0,0035 & 0,0241 \\
\hline 2006 & 0,0028 & 0,0307 & $-0,6194$ & 3,1418 & $-0,0177$ & 0,0077 & 0,0235 \\
\hline 2007 & 0,0048 & 0,0357 & $-0,7141$ & 3,7159 & $-0,0060$ & 0,0045 & 0,0282 \\
\hline Total & 0,0036 & 0,0343 & $-0,4943$ & 3,0665 & $-0,0156$ & 0,0059 & 0,0265 \\
\hline
\end{tabular}

DP: desvio padrão; Assim.: assimetria; Curt.: curtose; qt.: quartil, Med.: mediana.

Fonte: Elaborada pelos autores com base nos resultados da pesquisa.

A fim de testar a normalidade da série de taxas de retorno da carteira de mercado, foi empregado o teste de Doornik e Hansen (I994) implementado em Stata por Baum e Cox (200I). Foi obtida uma estatística $\chi_{2}^{2}=10,64$, o que implica a rejeição da hipótese nula de normalidade no nível de ı\% de significância. Um teste de normalidade particularmente importante para os propósitos desta pesquisa é o teste de assimetria e curtose de D’Agostino, Belanger e D’Agostino Jr. (I990). Por meio do resultado desse teste, é possível perceber qual momento estatístico é o responsável pela rejeição da hipótese nula de normalidade da variável. Quando aplicado à série de taxas de retorno do Ibovespa, verificou-se que a assimetria era o momento responsável pela rejeição da normalidade, e não a curtose.

Além disso, todas as empresas da amostra foram submetidas aos testes de normalidade sugerido por D’Agostino, Belanger e D'Agostino Jr. (I990). Os resultados mostraram que $5 \mathrm{I} \%$ das empresas da amostra apresentaram nível de significância observado inferior a 5\% para a assimetria. O nível de significância observado para a curtose foi inferior a $5 \%$ em $63 \%$ dos casos. No geral, considerando o teste conjunto dos dois momentos, a normalidade foi rejeitada em $68 \%$ das vezes, indicando, assim, que a maioria das empresas apresenta distribuição de probabilidade diferente da normal.

As empresas que compõem a amostra desta pesquisa são provenientes de diferentes setores da economia, com predominância de empresas dos setores de energia elétrica (II,7\%) e telecomunicações (IO,I\%). As estatísticas descritivas das variáveis de controle estão apresentadas na Tabela 2. Em média, o valor de 
mercado das companhias é aproximadamente o dobro do seu valor contábil, o índice de negociabilidade médio é de aproximadamente o,30 e o índice de endividamento médio é 0,47 .

TABELA 2

ESTATISTICAS DESCRITIVAS DAS VARIÁVEIS DE CONTROLE

\begin{tabular}{cccccc}
\hline VARIÁVEL & MÉDIA & DP & 10 QUARTIL & MEDIANA & 3o QUARTIL \\
\hline In RL & 14,094 & 1,462 & 12,970 & 14,055 & 15,260 \\
\hline VMVC & 1,999 & 1,440 & 0,840 & 1,670 & 2,805 \\
\hline NEG & 0,304 & 0,425 & 0,009 & 0,070 & 0,448 \\
\hline DBVM & 0,470 & 0,637 & 0,028 & 0,202 & 0,592 \\
\hline
\end{tabular}

Fonte: Elaborada pelos autores com base nos resultados da pesquisa.

A estimação dos comomentos seguiu o procedimento descrito na seção 3.I. O modelo utilizado, chamado modelo de mercado cúbico, é dado pela seguinte equação:

$$
r_{i}-r_{f} i=\alpha_{i}+\beta_{i}\left(r_{M}-r_{f}\right)+\gamma_{i}\left(r_{M}-r_{f}\right)^{2}+\delta_{i}\left(r_{M}-r_{f}\right)^{3}+\varepsilon_{i}
$$

tal que $\beta_{i}, \gamma_{i}$ e $\delta_{i}$ são, respectivamente, os vetores de dimensão $N \times$ I de covariâncias, coassimetrias e cocurtoses.

A Tabela 3 traz as informações acerca dos comomentos estimados pela primeira etapa do método empírico utilizado. A estimação foi feita pelo método dos mínimos quadrados ordinários (MQO).

\section{TABELA 3}

\section{ESTATÍSTICAS DESCRITIVAS DOS COMOMENTOS}

\begin{tabular}{llccccc}
\hline & COMOMENTO & MÉDIA & DP & 1ㅇ QUARTIL & MEDIANA & 3o QUARTIL \\
\hline$\beta$ & Covariância & 0,598 & 0,736 & 0,222 & 0,585 & 0,975 \\
$\gamma$ & Coassimetria & $-0,978$ & 8,132 & $-4,694$ & $-0,727$ & 2,287 \\
\hline$\delta$ & Cocurtose & 11,868 & 190,540 & $-59,432$ & 9,727 & 85,572 \\
\hline
\end{tabular}

Fonte: Elaborada pelos autores com base nos resultados da pesquisa. 


\subsection{RESULTADOS PRINCIPAIS}

O principal objetivo desta pesquisa é testar um modelo de apreçamento de ativos que utiliza comomentos como regressores. Desde o CAPM de Sharpe (I964) e Lintner (i965), modelos de apreçamento se utilizam de momentos estatísticos como medidas de risco. A metodologia mais usual neste tipo de pesquisa era a regressão linear simples, utilizando o método dos mínimos quadrados ordinários (MQO) ou algum outro mais robusto como o método dos momentos generalizado (GMM). Contudo, a natureza das séries financeiras é claramente uma série de dados no tempo, mas que também pode ser observada para diferentes empresas. Apesar disso, a análise de dados em painel, técnica estatística que combina dados observados tanto na dimensão transversal como na dimensão longitudinal, não havia sido utilizada, até onde sabemos, para testar modelos de apreçamento baseados em comomentos.

Após a estimação dos comomentos, conforme descrito pela Equação (I2), o próximo passo foi testar a significância deles em explicar o comportamento das taxas de retorno dos ativos. O modelo básico estimado foi o seguinte:

$$
\gamma_{i, t}=\theta_{\mathrm{o}}+\theta^{\mathrm{T}} \Theta_{i, t}+\omega^{\mathrm{T}} \Omega_{i, t}+\varepsilon_{i, t}, i=\mathrm{I}, \ldots, N \text { e } t=\mathrm{I}, \ldots, \mathrm{T}
$$

tal que, para cada empresa i em determinado ano $t$, foi observado um conjunto $k_{\mathrm{I}}$ de comomentos representados em $\Theta_{i, t}$, um vetor de dimensão $k_{\mathrm{I}} \times \mathrm{I}$. O vetor $\theta^{\mathrm{T}}$, de dimensão $k_{\mathrm{I}} \times \mathrm{I}$, de parâmetros associados a cada comomento, transposto. O conjunto das $k_{2}$ variáveis de controle está representado no vetor $\Omega_{i, t}$. O vetor $\omega^{\mathrm{T}}$ de dimensão $k_{2} \times \mathrm{I}$, transposto, é o conjunto de parâmetros das variáveis de controle. O termo de erro, $\varepsilon_{i, t}$, não deve, por hipótese, estar correlacionado contemporaneamente com os regressores; e a variável de resposta, $\gamma_{i, t}$, é o prêmio pelo risco da empresa $i$ no instante de tempo $t$.

As variáveis de controle empregadas nesta pesquisa foram escolhidas a partir de resultados verificados em estudos empíricos anteriores. Os controles utilizados foram: tamanho da empresa, valor de mercado sobre valor contábil das ações, índice de negociabilidade, índice de alavancagem, setor de atividade e dummies de ano. A variável que servia de proxy para tamanho das empresas causou problemas de multicolinearidade nos modelos estimados, principalmente por conta da presença das dummies de ano. O problema desaparecia quando se excluía da estimação a variável de tamanho ou o conjunto de dummies de ano. Decidiu-se por manter as dummies de ano como regressoras no modelo e, para não perder completamente o conteúdo informacional contido na variável de tamanho, utilizá-las como variável instrumental. Foram testadas outras proxies para tamanho, como valor total dos ativos e valor de mercado das ações, porém não houve sucesso na eliminação do problema de multicolinearidade. 
Outro aspecto metodológico tratado pela investigação empírica foi com relação às observações extremas. Empregou-se a técnica de "winsorização" das variáveis conforme procedimento apresentado na Seção 3.4.

Os parâmetros da Equação (13) poderiam ser estimados por diferentes métodos, como o método dos mínimos quadrados ordinários agrupados (Pols). Contudo, esse método não considera a heterogeneidade não observada, ou seja, não captura características das empresas invariantes no tempo e que podem influenciar o comportamento da variável dependente. A fim de considerar a heterogeneidade, o modelo pode incluir um intercepto para cada empresa, igual para todos os instantes de tempo. O painel pode ser estimado com efeitos fixos ou aleatórios. O EA supõe que a correlação entre as variáveis explicativas e o efeito não observado é nula. Já o EF permite que essa correlação seja não nula. Ambos os procedimentos, EF e EA, adotam o pressuposto de exogeneidade estrita dos regressores. Para verificar essa condição, foram aplicados os testes de exogeneidade estrita conforme as equações (8) e (9). Os resultados dos testes apontam para a rejeição da hipótese nula de que os regressores são estritamente exógenos. Como consequência desse resultado, deve ser utilizado o GMM como método de estimação, uma vez que ele lida adequadamente com o problema de variáveis independentes endógenas.

O modelo proposto foi estimado por quatro distintos métodos: GMM-Difi, GMM-Dif2, GMM-Sisı e GMM-Sis2. As estimações por GMM são apresentadas nas versões em um e dois estágios. Para detalhes sobre a diferença entre cada uma dessas versões, ver Roodman (2009). Para os modelos estimados em dois estágios, foi aplicada a correção proposta por Windmeijer (2005) com o objetivo de reduzir problemas com viés no cálculo dos erros padrão em amostras finitas. Assim, a nomenclatura GMM-Difi significa que o GMM em diferenças foi estimado em um estágio. Raciocínio análogo se aplica aos demais casos.

Espera-se que o sinal do coeficiente associado à variável $\beta$ seja positivo, ou seja, quanto maior a covariância entre as taxas de retorno do ativo e da carteira de mercado, maior a taxa de retorno esperada do ativo. O sinal esperado do coeficiente associado à variável $\gamma$, a coassimetria, é negativo, ou seja, quanto maior a coassimetria entre as taxas de retorno do ativo e da carteira de mercado, menor a taxa de retorno esperada do ativo. E, por fim, o sinal esperado do coeficiente associado à variável cocurtose é positivo, ou seja, os investidores esperam taxas de retorno maiores para os ativos que apresentam maior cocurtose entre as taxas de retorno do ativo e da carteira de mercado.

As variáveis de controle têm os seguintes sinais esperados:

- A alavancagem deveria apresentar sinal positivo, pois empresas mais endividadas são vistas como mais arriscadas e, portanto, deveriam proporcionar maiores prêmios aos investidores. 
- O valor de mercado sobre valor contábil, se apresentar sinal positivo, indicará que os investidores veem as empresas com elevado índice como mais arriscadas, talvez por consequência da maior dificuldade de avaliação dos fundamentos dessas empresas (maior intangibilidade); se apresentar sinal negativo, indicará que os investidores veem as empresas com alto índice como capazes de gerar mais valor a partir de um mesmo montante de ativos, e, por consequência, aceitariam um prêmio mais baixo.

- O índice de negociabilidade deveria apresentar sinal negativo, uma vez que empresas com alta negociabilidade têm mais chances de ser negociadas pelo seu preço justo, o que implica que investidores aceitariam um prêmio mais baixo por essas empresas.

Os resultados das estimações do modelo, apresentados na Tabela 4, indicam que o coeficiente do comomento $\beta$ é positivo e significante em todas as estimações, sendo significante no nível de Io\% no GMM-Sisi, no nível de 5\% no GMM-Difr e GMM-Sis2, e no nível de I\% para o modelo estimado por GMM-Dif2. A coassimetria não apresentou coeficiente significativo em nenhuma das estimações. Já a cocurtose se mostrou positiva e significante em todas as estimações, sendo significante no nível de Io\% quando estimado por GMM-Sis2, significante no nível de 5\% quando estimado por GMM-Dif2 e GMM-Sisi e significante no nível de ı\% quando estimado por GMM-Difi.

A variável valor de mercado sobre valor contábil (VMVC) se mostrou positiva e significante no nível de Io\% nas estimações por GMM-Sis. O sinal positivo indica que os investidores veem as empresas com alto VMVC como mais arriscadas e, por isso, exigem um prêmio mais elevado. A variável de endividamento não se mostrou significante em nenhum dos modelos, e o índice de negociabilidade, na estimação do GMM sistêmico em dois estágios, mostrou-se negativo e significante, conforme esperado, no nível de ı०\%.

Os modelos de dois estágios foram estimados com a correção proposta por Windmeijer (2005) para amostras finitas. As análises de diagnóstico, reportadas ao final da Tabela 4, apontam em favor das hipóteses de identificação: as estatísticas $m_{\mathrm{I}}$, que medem as autocorrelações de primeira ordem nas diferenças, são negativas e significantes, e as estatísticas $m_{2}$, que medem as autocorrelações de segunda ordem, não são significantes. Além disso, para a estimação do GMM sistêmico, não foi possível rejeitar a hipótese nula do teste J de Hansen. Isso significa que os instrumentos utilizados não são correlacionados com os erros e podem ser utilizados no modelo. Já para as estimações por GMM em diferenças, esse resultado não se mostrou satisfatório. O teste de DIF-Hansen não rejeitou a hipótese nula, validando as restrições adicionais requeridas pelo GMM sistêmico. Portanto, a estimação do modelo por GMM-Sis é mais indicada. 
TABELA 4

RESULTADOS DAS ESTIMAÇÕES

\begin{tabular}{|c|c|c|c|c|}
\hline & GMM-DIF1 & GMM-DIF2 & GMM-SIS1 & GMM-SIS2 \\
\hline$\beta$ & $0,416^{* *}(0,129)$ & $0,459 * * *(0,139)$ & $0,305^{*}(0,131)$ & $0,387 * *(0,130)$ \\
\hline$\gamma$ & $-0,008(0,006)$ & $0,000(0,007)$ & $-0,009(0,006)$ & $0,001(0,007)$ \\
\hline$\delta$ & $0,001 * * *(0,000)$ & $0,001 * *(0,000)$ & $0,001 * *(0,000)$ & $0,001 *(0,000)$ \\
\hline VMVC & $0,035(0,023)$ & $0,036(0,031)$ & $0,020 *(0,009)$ & $0,023 *(0,011)$ \\
\hline DBVM & $-0,015(0,014)$ & $-0,007(0,019)$ & $0,021(0,017)$ & 0,022 (0,038) \\
\hline NEG & $-0,075(0,043)$ & $-0,083(0,065)$ & $-0,043(0,024)$ & $-0,054^{*}(0,026)$ \\
\hline Qtde. obs. & 406 & 406 & 585 & 585 \\
\hline Qtde. instrum. & 23 & 23 & 46 & 46 \\
\hline VIF máximo & \multicolumn{2}{|c|}{4,63} & \multicolumn{2}{|c|}{6,36} \\
\hline$m_{1}$ & $-4,433(0,000)$ & $-4,085(0,000)$ & $-4,403(0,000)$ & $-3,980(0,000)$ \\
\hline$m_{2}$ & $-1,236(0,216)$ & $-1,218(0,223)$ & $-1,020(0,308)$ & $-1,289(0,197)$ \\
\hline J de Hansen & \multicolumn{2}{|c|}{$23,457(13 ; 0,037)$} & \multicolumn{2}{|c|}{$23,439(17 ; 0,135)$} \\
\hline DIF-Hansen & & & \multicolumn{2}{|c|}{$6,85(4 ; 0,144)$} \\
\hline
\end{tabular}

Nota: A variável dependente é o prêmio pelo risco das ações. Esta e os regressores foram definidos na seção 3.5. Os estimadores utilizados foram o GMM em diferenças e o GMM sistêmico de um e dois estágios, com transformações de desvios ortogonais. Como instrumentos, utilizaram-se as transformações de primeiras diferenças e as defasagens dos comomentos e da alavancagem, e as transformações de primeiras diferenças do logaritmo natural da receita líquida. Assume-se que os demais regressores são estritamente exógenos. Os erros padrão (valores dentro dos parênteses) foram obtidos utilizando dados agrupados por empresa, de maneira robusta a quaisquer formas de heteroscedasticidade e/ou autocorrelação. Os símbolos *, *** e **** denotam, respectivamente, a significância estatística nos níveis de I0\%, 5\% e I\%. Valores maiores que io para a estatística VIF (variance inflation factor) indicam problemas de multicolinearidade nos regressores. As estatísticas $\mathrm{m}_{\mathrm{I}}$ e $\mathrm{m}_{2}$ referem-se aos testes de autocorrelação de primeira e segunda ordens. Para o teste de Hansen e DIF-Hansen, reportam-se a estatística de teste e, entre parênteses, o número de graus de liberdade e seu nível de significância observado, respectivamente. Para os testes de autocorrelação, reportam-se as estatísticas de teste $\left(\mathrm{m}_{\mathrm{I}}\right.$ e $\left.\mathrm{m}_{2}\right)$ e, entre parênteses, o nível de significância observado correspondente.

Fonte: Elaborada pelos autores com base nos resultados da pesquisa. 
Alternativamente, diferentes especificações do modelo foram testadas como forma de garantir a robustez dos resultados encontrados. Especificamente, foram empregadas variáveis regressoras após o processo de "winsorização" conforme descrito na Seção 3.4. Os resultados encontrados não diferiram dos resultados principais, exceto algumas vezes, com relação ao nível de significância observado. As magnitudes dos coeficientes dos comomentos, todavia, não sofreram alterações qualitativas, mostrando que os resultados são robustos à eventual presença de observações extremas. Para fins de comparação dos resultados, foram estimados o CAPM tradicional (modelo A), o CAPM com variáveis de controle (modelo C) e um modelo com comomentos sem controles (modelo B). Esses resultados encontram-se na Tabela 5, e todos foram estimados por GMM-Sis em dois estágios. Os resultados não apresentaram diferenças qualitativamente relevantes quando estimados por GMM-Dif ou com apenas um estágio.

\section{TABELA 5}

RESULTADO DAS ESTIMAÇÕES ALTERNATIVAS

\begin{tabular}{|c|c|c|c|}
\hline & MODELO A & MODELO B & MODELO C \\
\hline$\beta$ & $0,123 *(0,068)$ & $0,398 * * *(0,114)$ & $0,159 * *(0,069)$ \\
\hline$\gamma$ & & $-0,004(0,006)$ & \\
\hline$\delta$ & & $0,001 * * *(0,000)$ & \\
\hline VMVC & & & $0,021 * *(0,010)$ \\
\hline DBVM & & & $0,023(0,039)$ \\
\hline NEG & & & $-0,029 * *(0,013)$ \\
\hline Qtde. obs. & 621 & 621 & 585 \\
\hline Qtde. instrum. & 28 & 38 & 36 \\
\hline VIF máximo & 2,40 & 5,15 & 2,45 \\
\hline$m_{1}$ & $-4,371(0,000)$ & $-4,355(0,000)$ & $-3,977(0,000)$ \\
\hline$m_{2}$ & $-1,212(0,226)$ & $-1,572(0,116)$ & $-1,415(0,157)$ \\
\hline J de Hansen & $4,475(4 ; 0,345)$ & $22,606(12 ; 0,031)$ & $12,797(9 ; 0,172)$ \\
\hline DIF-Hansen & $0,17(1 ; 0,678)$ & $6,43(3 ; 0,092)$ & $3,72(2 ; 0,156)$ \\
\hline
\end{tabular}

Nota: A variável dependente é o prêmio pelo risco das ações. Esta e os regressores foram definidos na seção 3.5. Os coeficientes foram estimados por GMM sistêmico de dois estágios, com transformações de desvios ortogonais. Como instrumentos, utilizaram-se as transformações de primeiras 
diferenças e as defasagens dos comomentos (beta nos modelos A e C, e todos os comomentos no modelo B) e da alavancagem (modelo C), e as transformações de primeiras diferenças do logaritmo natural da receita líquida (modelo C). Assume-se que os demais regressores são estritamente exógenos. Os erros padrão (valores dentro dos parênteses) foram obtidos utilizando dados agrupados por empresa, de maneira robusta a quaisquer formas de heteroscedasticidade e/ou autocorrelação. Os símbolos *, ** e $* * *$ denotam, respectivamente, a significância estatística nos níveis de I०\%, $5 \%$ e I\%. Valores maiores que io para a estatística VIF (variance inflation factor) indicam problemas de multicolinearidade nos regressores. O teste da estatística J de Hansen é uma versão robusta do teste de sobreidentificação de Sargan. As estatísticas $m_{I}$ e $m_{2}$ referem-se aos testes de autocorrelação de primeira e segunda ordens. O DIF-Hansen baseia-se na diferença entre as estatísticas de Hansen e Sargan calculadas para o mesmo modelo estimado por GMM-Sis e GMM-Dif, ambos de dois estágios. Para o teste de Hansen e DIF-Hansen, reportam-se a estatística de teste e, entre parênteses, o número de graus de liberdade e seu nível de significância observado, respectivamente. Para os testes de autocorrelação, reportam-se as estatísticas de teste $\left(m_{I}\right.$ e $\left.m_{2}\right)$ e, entre parênteses, o nível de significância observado correspondente.

Fonte: Elaborada pelos autores com base nos resultados da pesquisa.

O modelo A foi estimado apenas com o beta original do CAPM, que se mostrou positivo e significante no nível de ı०\%. O modelo B, que utilizou todos os comomentos, apresentou resultados que não diferiram dos encontrados na especificação original, ou seja: a covariância e a cocurtose se mostraram significantes, desta vez no nível de I\%, e a coassimetria não se mostrou significante. O modelo $\mathrm{C}$, em relação ao modelo $\mathrm{A}$, acrescentou as variáveis de controle. O beta se mostrou positivo e significante no nível de $5 \%$, o valor de mercado sobre o valor contábil se mostrou positivo e significante no nível de $5 \%$, o índice de negociabilidade se mostrou negativo e significante no nível de $5 \%$, e a alavancagem não se mostrou significante. Assim, todos os resultados apresentados apontam para a mesma conclusão: a de que fatores de risco baseados em comomentos adicionam informação a respeito do comportamento das taxas de retorno das ações.

\section{CONCLUSÃO}

O CAPM tradicional, desenvolvido por Sharpe (I964) e Lintner (I965), é largamente utilizado em estudos financeiros para determinar, em termos teóricos, a taxa de retorno exigida de um ativo ou carteira de ativos, fazendo uso de uma única medida de risco sistêmico. Apoiando-se em uma série de pressupostos, esse modelo foi amplamente testado em estudos prévios, e os resultados, quando confrontados, podem ser caracterizados como ambíguos. Além disso, algumas observações empíricas levaram os pesquisadores a sugerir modificações no modelo. Nas palavras de Barone-Adesi, Gagliardini e Urga (2004): 
Embora a persistência dessas anomalias ao longo do tempo ainda seja controversa, a evidência sugere que o CAPM de média-variância não é uma descrição satisfatória do equilíbrio de mercado. Essas anomalias de apreçamento podem estar relacionadas à possibilidade de que fatores inúteis estejam sendo apreçados. Claro, também é possível que as anomalias de apreçamento se devam a fatores omitidos.

Outro aspecto muito debatido e testado empiricamente é a questão da distribuição da taxa de retorno dos ativos. A violação dessa hipótese pode tornar a estimação dos parâmetros de interesse enviesada, implicando decisões incorretas a respeito do teste das hipóteses derivadas das argumentações teóricas das pesquisas. Segundo Brooks et al. (2005), a distribuição não normal das taxas de retorno dos ativos é um fato estilizado aceito quase universalmente. Conforme Silva (2005):

Sem normalidade, é improvável que o CAPM se sustente, mas, quando os retornos são normais, nós só precisamos da média e da variância para descrever perfeitamente a distribuição. No entanto, um número infinito de momentos geralmente é requerido para especificar completamente as caudas de uma distribuição. Os investidores estão preocupados com o risco, e risco deve ser medido em termos da distribuição de probabilidade completa, o que, por sua vez, pode ser medido com os momentos da distribuição. Só em casos muito especiais, tais como a utilidade quadrática ou normalidade dos retornos, podemos ignorar os momentos de ordem mais alta e comomentos.

Se, por um lado, estudos como os de Fama e French (1992, 1993) argumentam que outros fatores como o tamanho e valor das empresas devem ser considerados em modelos de apreçamento, há, por outro, estudos que argumentam que o risco sistêmico medido pelo beta não deveria ser o único presente no modelo. Autores como Rubinstein (I973), Kraus e Litzenberger (1976), Fang e Lai (I997), entre outros, sugerem formulações de modelos que levam em consideração riscos sistêmicos relacionados à assimetria e à curtose.

A pesquisa desenvolvida neste artigo faz uso dos comomentos baseados na variância, assimetria e curtose das taxas de retorno dos ativos e da carteira de mercado como regressores em um modelo de apreçamento. Além destes, outros fatores são reportados na literatura como responsáveis pelo aparecimento de relações ditas anômalas. A fim de controlá-los, tais variáveis foram incluídas no modelo sob a forma de controles. A intenção, evidentemente, foi verificar empiricamente se, com dados de empresas brasileiras não financeiras, usando 
uma abordagem metodológica robusta, os comomentos seriam estatisticamente significantes.

Especificamente, o interesse da pesquisa recaiu sobre as empresas mais líquidas negociadas na BM\&FBovespa no período de 2003 a 2007. Empresas com baixa liquidez foram excluídas da amostra porque a determinação verdadeira de seu preço fica prejudicada, dado que a ocorrência de negociação dos seus papéis é baixa. Além dessas empresas, empresas do setor financeiro também foram excluídas, uma vez que apresentam características muito particulares, que as fazem diferir sobremaneira das demais empresas pertencentes a outros setores de atividade.

A importância deste estudo também reside no fato de serem usados dados de um mercado emergente como o Brasil para realizar a pesquisa. Estudos que utilizam dados americanos para testar a relevância estatística dos comomentos em modelos de apreçamento estão se tornando, aos poucos, comuns. No entanto, existem poucos estudos realizados com dados de outros países. No Brasil, especificamente, modelos semelhantes foram testados por Silva (2005) e Ceretta, Catarina e Muller (2007), porém em uma abordagem metodologicamente distinta da desta pesquisa.

É especialmente importante considerar alguns aspectos relevantes aos mercados emergentes, especialmente o brasileiro. Conforme Bekaert et al. (I998),

[...] a análise padrão baseada em média-variância é um pouco problemática no que diz respeito aos mercados emergentes. Nesta análise, os investidores se preocupam com os retornos esperados, variâncias e covariâncias, mas os retornos de mercados emergentes não podem ser completamente caracterizados por apenas essas medidas. [...] há significativa assimetria e curtose nesses retornos.

A metodologia tradicionalmente empregada nos testes empíricos de modelos de apreçamento (CAPM original ou variações deste) costuma negligenciar o efeito conjunto que a característica transversal e longitudinal dos dados pode proporcionar. Esta pesquisa, além de empregar variáveis de controle criadas a partir de anomalias relatadas na literatura, fez uso de uma abordagem metodológica com dados em painel estimados por GMM para testar a significância dos comomentos. Essa verificação empírica, até onde sabemos, é inédita na literatura, constituindo, assim, uma das principais contribuições do trabalho.

Uma primeira verificação dos dados de taxas de retorno das empresas que fazem parte da amostra da pesquisa mostrou que, de fato, a normalidade não é comum. Foram aplicados dois testes distintos a fim de testar a hipótese de normalidade, e a maioria das empresas apresentou distribuição não normal. A carteira de mercado também foi submetida aos mesmos testes e teve a hipótese nula 
de normalidade distribucional rejeitada. Especificamente, a carteira de mercado teve a assimetria considerada distante da assimetria de uma normal. A curtose, no entanto, se encontrava dentro do esperado para uma distribuição normal. O mesmo resultado não foi observado nos ativos, em que a curtose foi rejeitada mais vezes que a assimetria.

Os resultados mostraram que os comomentos $\beta$ e $\delta$, respectivamente, a covariância e a cocurtose entre as taxas de retorno dos ativos e da carteira de mercado, são significantes nos modelos estimados. O comomento $\beta$ se mostrou significante no nível de I०\% quando estimado por GMM-Sis em um estágio, significante no nível de 5\% quando estimado por GMM-Dif em um estágio e GMM-Sis em dois estágios e no nível de $\mathrm{I} \%$ quando estimado por GMM-Dif em dois estágios. Já o comomento $\delta$ foi significante no nível de I०\% no modelo estimado por GMM-Sis em dois estágios, no nível de $5 \%$ quando estimado por GMM-Dif em dois estágios e GMM-Sis em um estágio e no nível de I\% quando estimado por GMM-Dif em um estágio. Os resultados não se alteraram de maneira significativa quando variáveis "winsorizadas" foram utilizadas nas estimações ou mesmo quando se empregaram especificações alternativas.

Em síntese, esta pesquisa proporcionou a primeira evidência com dados brasileiros de um modelo de apreçamento baseado em comomentos, controlando as anomalias comumente reportadas na literatura e com dados em painel estimados por GMM. Os resultados permitem afirmar que a formulação clássica do CAPM é incompleta ao não considerar outros fatores de risco como relevantes para a determinação do preço dos ativos. As evidências são consistentes com outros estudos já realizados em outros países, porém a comparação fica comprometida, uma vez que o método empregado é bastante distinto. Pesquisas futuras poderão ser feitas explorando ainda mais esse tema. De maneira prática, é possível explorar os efeitos de outros comomentos, como os de ordem superior à cocurtose ou ainda empregar outras variáveis como controle. Conforme Chung, Johnson e Schill (2006), há indícios de que o aumento da quantidade de comomentos no modelo de apreçamento diminui a relevância estatística de fatores de risco como os propostos por Fama e French (1992, I993).

\section{COSKEWNESS, COKURTOSIS AND STOCK RATES OF RETURN: A PANEL DATA ANALYSIS}

\section{ABSTRACT}

Asset pricing models have been a constant theme in finance research. Since the Capital Asset Pricing Model (CAPM) proposed by Sharpe (I964), such models 
linearly relate the expected rate of returns of an asset or portfolio of assets with systemic risk factors. This research presents a test of a pricing model with risk factors based on statistical comoments and using a Brazilian dataset. The proposed model is an extension of the original CAPM with the addition of coskewness and cokurtosis between stock rates of return and the market portfolio rates of return. The effects of other variables such as market to book value, financial leverage and a negotiability index served as control variables. The sample consisted of I79 non-financial Brazilian companies traded on BM\&FBovespa with data available from 2003 to 2007 . Annual systemic moments were calculated from weekly rates of return. They were then tested on a pricing model in order to check for the existence of a risk premium associated with each of these risk measures. We employed a Generalized Method of Moments (GMM) panel data analysis. The use of GMM aims to address potential problems of endogeneity and simultaneous determination of the data, avoiding the occurrence of bias in the estimates. The estimation results show that the relationship between rates of return and covariance and cokurtosis are statistically significant. The results were robust to alternative model specifications. This research contributes to the literature by presenting empirical evidence that there is a Brazilian risk premium associated with systemic moments.

\section{KEYWORDS}

Coskewness; Cokurtosis; Rates of return; Panel data; GMM.

\section{COASSIMETRIA, COCURTOSE Y RENDIMIENTOS DE LAS ACCIONES: COM ANÁLISIS COM DATOS DE PANEL}

\section{RESUMEN}

Modelos de fijación de activos han sido un tema constante en la investigación de finanzas. Desde el modelo de fijación de precios de activos de capital (CAPM) propuesto por Sharpe (I964) se relacionan, generalmente en forma lineal, la tasa de retorno esperada de un activo o cartera de activos con factores de riesgo sistémico. Esta investigación presenta una prueba de un modelo de precios con los datos de Brasil y factores de riesgo com el apoyo de comomentos estadísticos. El modelo propuesto es una extensión del CAPM original más la cocurtose y 
la coassimetria entre las tasas de rendimiento de las acciones de las empresas que componen la muestra y las tasas de retorno de la cartera de mercado. Los efectos de otras variables como el valor de mercado sobre el valor en libro, el apalancamiento financiero y un índice de comerciabilidad bursátil como variables de control. La muestra estuvo conformada por I79 empresas no financieras brasileñas que cotizan en el BM\&FBovespa y con los datos disponibles entre los años 2003 a 2007 . La metodología consistió en calcular los momentos sistémicos anuais a partir de las tasas de rendimiento semanais y luego ponerlos a prueba en un modelo de precios con el fin de comprobar si hay una prima de riesgo asociados a cada una de estas medidas de riesgo. Empleamos um técnica de análisis de datos de panel calculada por el método generalizado de momentos (GMM). El uso del método generalizado de momentos tiene como objetivo abordar los posibles problemas de endogeneidad y determinación simultánea de los datos, evitando la aparición de sesgos en las estimaciones. Los resultados de la estimación indican que la relación de las tasas de retorno de los activos y la covarianza y cocurtose son estadísticamente significativas. Los resultados fueron robustos a especificaciones alternativas del modelo. Esta investigación contribuye a la literatura mediante la presentación de evidencia empírica de que hay una prima de riesgo asociado con momentos sistémicos.

\section{PALABRAS CLAVE}

Coassimetria; Cocurtose; Rendimientos de las acciones; Datos de panel; GMM.

\section{REFERÊNCIAS}

ARDITTI, F. D. Risk and the required return on equity. The Journal of Finance, v. 22, n. I, p. I9-36, 1967.

ARELLANO, M.; BOND, S. Some tests of specification for panel data: Monte Carlo evidence and an application to employment equations. The Review of Economic Studies, v. 58, n. I94, p. 277-297, I99I.

ARELLANO, M.; BOVER, O. Another look at the instrumental variable estimation of error-components models. Journal of Econometrics, v. 68, n. I, p. 29-52, I995.

BALTAGI, B. H. Economic analysis of panel data. 3. ed. Chichester: Wiley, 2005.

Panel data models. In: MILLS, T. C.; PATTERSON K. Palgrave handbook of econometrics. New York: Palgrave Macmillan, 2006. p. 633-66I.

BANZ, R. W. The relationship between return and market value of common stocks. Journal of Financial Economics, v. 9, n. I, p. 3-18, I98I.

BARNETT, V.; LEWIS, T. Outliers in statistical data. 3. ed. Chichester: Wiley, I994. 
BARONE-ADESI, G.; GAGLIARDINI, P.; URGA, G. Testing asset pricing models with coskewness. Journal of Business \& Economic Statistics, v. 22, n. 4, p. 474-486, 2004.

BAUM, C. F.; COX, N. J. Omninorm: stata module to calculate omnibus test for univariate/multivariate normality. Statistical Software Components. Boston: Boston College Department of Economics, 200I. Disponível em: <http://ideas.repec.org/c/boc/bocode/s4I75or.html>. Acesso em: 4 maio 2008.

BAUM, C. F.; SCHAFFER, M. E.; STILLMAN, S. Instrumental variables and GMM: estimation and testing. Stata Journal, v. 3, n. I, p. I-3I, 2003.

BEKAERT, G. et al. Distributional characteristics of emerging market equities and asset allocation. Journal of Portfolio Management, v. 24, n. 2, p. I02-II6, I998.

BERK, J. B. A critique of size-related anomalies. Review of Financial Studies, v. 8, n. 2, p. 275-286, I995.

BHANDARI, L. C. Debt/equity ratio and expected common stock returns: empirical evidence. The Journal of Finance, v. 43, n. 2, p. 507-528, I988.

BLACK, F. S. Capital market equilibrium with restricted borrowing. The Journal of Business, v. 45, n. 3, p. 444-455, I972.

BLACK, F. S.; JENSEN, M. C.; SCHOLES, M. The capital asset pricing model: some empirical tests. In: JENSEN, C. (Ed.). Studies in the Theory of Capital Markets. New York: Praeger Publishers Inc., I972. p. 79-I20.

BLUNDELL, R.; BOND, S. R. Initial conditions and moment restrictions in dynamic panel data models. Journal of Econometrics, v. 87, n. I, p. II5-I43, I998.

BOND, S. R.; HOEFFLER, A.; TEMPLE, J. R. GMM estimation of empirical growth models. SSRN eLibrary, 200I. Disponível em: <http://ssrn.com/paper=290522>. Acesso em: I set. 2009.

BROOKS, C. et al. Autoregressive conditional kurtosis. Journal of Financial Econometrics, v. 3, n. 3, p. 399, 2005 .

CERETTA, P. S.; CATARINA, G. F. S.; MULLER, I. Modelo de precificação incorporando assimetria e curtose sistemática. In: ENANPAD, 3I., 2007, Rio de Janeiro. Anais... Rio de Janeiro: Anpad, 2007.

CHUNG, Y. P.; JOHNSON, H.; SCHILL, M. J. Asset pricing when returns are nonnormal: FamaFrench factors versus higher order systematic comoments. Journal of Business, v. 79, n. 2, p. 923$940,2006$.

D’AGOSTINO, R. B.; BELANGER, A.; D'AGOSTINO JR., R. B. A suggestion for using powerful and informative tests of normality. The American Statistician, v. 44, n. 4, p. 3I6-32I, I990.

DOORNIK, J. A.; HANSEN, H. An omnibus test for univariate and multivariate nor-mality. Nuffield College, Oxford University, I994. Disponível em: <http://ideas.uqam.ca/ideas/data/ Papers/wuknucowp9604.html>. Acesso em: 4 maio 2008.

FAMA, E. F.; FRENCH, K. R. The cross-section of expected stock returns. The Journal of Finance, v. 47 , n. 2, p. 427-465, I992.

. Common risk factors in the returns on stocks and bonds. Journal of Financial Economics,

v. 33, p. 3-56, I993.

FAMA, E. F.; MACBETH, J. D. Risk, return, and equilibrium: empirical tests. The Journal of Political Economy, v. 8I, n. 3, p. 607-636, I973.

FANG, H.; LAI, T. Y. Co-kurtosis and capital asset pricing. The Financial Review, v. 32, n. 2, p. 293307, I997. 
FOGLER, H. R.; RADCLIFFE, R. C. A note on measurement of skewness. The Journal of Financial and Quantitative Analysis, v. 9, n. 3, p. 485-489, I974.

FRIEND, I.; WESTERFIELD, R. Co-skewness and capital asset pricing. Journal of Finance, v. 35, n. 4, p. 897-9I3, I980.

GALAGEDERA, D. U. A.; HENRY, D.; SILVAPULLE, P. Empirical evidence on the conditional relation between higher-order systematic co-moments and security returns. Quaterly Journal of Business and Economics, v. 42, p. I2I-I37, 2003.

HANSEN, L. P. Large sample properties of generalized method of moments estimators. Econometrica, v. 50, n. 4, p. 1029-1054, I982.

HARVEY, C. R. et al. Portfolio selection with higher moments. Working Paper, 2004. Disponível em: <http://papers.ssrn.com/sol3/papers.cfm?abstract_id=634I4I >. Acesso em: 29 mar. 2008.

HARVEY, C. R.; SIDDIQUE, A. Conditional skewness in asset pricing tests. Journal of Finance, v. 55, n. 3, p. $1263-1295,2000$.

HAWAWINI, G. A. An analytical examination of the intervaling effect on skewness and other moments. The Journal of Financial and Quantitative Analysis, v. I5, n. 5, p. II2I-II27, I980.

HOMAIFAR, G.; GRADDY, D. B. Equity yields in models considering higher moments of the return distribution. Applied Economics, v. 20, n. 3, p. 325-334, I988.

JAGANNATHAN, R.; WANG, Z. The conditional CAPM and the cross-section of expected returns. The Journal of Finance, v. 5I, n. I, p. 3-53, I996.

JONDEAU, E.; ROCKINGER, M. The allocation of assets under higher moments. Fame Research Paper Series n. rp7I, 2002. Disponível em: <http://ideas.repec.org/p/fam/rpseri/rp7i.html>. Acesso em: 29 mar. 2008.

Conditional volatility, skewness, and kurtosis: existence, persistence, and comovements.

Journal of Economic Dynamics and Control, v. 27, n. 10, p. 1699-1737, 2003.

. Optimal portfolio allocation under higher moments. European Financial Management, v. I2, n. I, 29-55, 2006.

KAN, R.; ZHANG, C. GMM tests of stochastic discount factor models with useless factors. Journal of Financial Economics, v. 54, n. I, p. 103-127, 1999a.

KAN, R.; ZHANG, C. Two-pass tests of asset pricing models with useless factors. The Journal of Finance, v. 54, n. I, p. 203-235, I999b.

KRAUS, A.; LITZENBERGER, R. H. Skewness preference and the valuation of risk assets. The Journal of Finance, v. 3I, n. 4, p. 1085-1100, I976.

LAU, H. S.; WINGENDER, J. R. The analytics of the intervaling effect on skewness and kurtosis of stock returns. The Financial Review, v. 24, n. 2, p. 216-233, I989.

LIM, K. G. A new test of the three-moment capital asset pricing model. The Journal of Financial and Quantitative Analysis, v. 24, n. 2, p. 205-215, I989.

LINTNER, J. The valuation of risk assets and the selection of risky investments in stock portfolios and capital budgets. The Review of Economics and Statistics, v. 47, n. I, p. 13-37, 1965.

MARKOWITZ, H. M. Portfolio selection. The Journal of Finance, v. 7, n. I, p. 77-91, I952.

Portfolio selection: efficient diversification of investments. New York: Wiley, I959.

MERTON, R. C. An intertemporal capital asset pricing model. Econometrica, v. 4I, n. 5, p. 867-887, I973.

ROODMAN, D. M. How to do xtabond2: an introduction to difference and system GMM in Stata. Stata Journal, v. 9, n. I, p. 86-136, 2009 . 
ROSENBERG, B.; REID, K.; LANSTEIN, R. Persuasive evidence of market inefficiency. Journal of Portfolio Management, v. II, n. 9, p. I7, I985.

ROSS, S. A. The arbitrage theory of capital asset pricing. Journal of Economic Theory, v. I3, n. 3, p. 34I-360, I976.

RUBINSTEIN, M. The fundamental theorem of parameter-preference security valuation. Journal of Financial and Quantitative Analysis, v. 8, p. 6I-69, I973.

SAITO, R.; BUENO, R. D. L. Fundamentos teóricos e empíricos de apreçamento de ativos. Revista de Administração de Empresas, v. 47, p. 8I-85, 2007.

SARGAN, J. D. The estimation of economic relationships using instrumental variables. Econometrica, v. 26, p. 393-415, I958.

SHANKEN, J. On the estimation of beta-pricing models. Review of Financial Studies, v. 5, n. I, p. I-55, I992.

SHARPE, W. F. Capital asset market prices: a theory of market equilibrium under conditions of risk. The Journal of Finance, v. I9, n. 3, p. 425-442, I964.

SILVA, A. L. C. Modeling and estimating a higher sistematic co-moment asset pricing model in the Brazilian stock market. Latin American Business Review, v. 6, p. 85-IOI, 2005.

SINGLETON, J. C.; WINGENDER, J. Skewness persistence in common stock returns. The Journal of Financial and Quantitative Analysis, v. 21, n. 3, p. 335-34I, I986.

SMITH, K. V. The effect of intervaling on estimating parameters of the capital asset pricing model. The Journal of Financial and Quantitative Analysis, v. I3, n. 2, p. 313-332, I978.

STATTMAN, D. Book values and stock returns. The Chicago MBA: a Journal of Selected Papers, v. 4 , p. $25-45$, I980.

WINDMEIJER, F. A finite sample correction for the variance of linear efficient two-step GMM estimators. Journal of Econometrics, v. I26, n. I, p. 25-51, 2005.

WOOLDRIDGE, J. M. Econometric analysis of cross section and panel data. Cambridge, MA: MIT Press, 2002. 